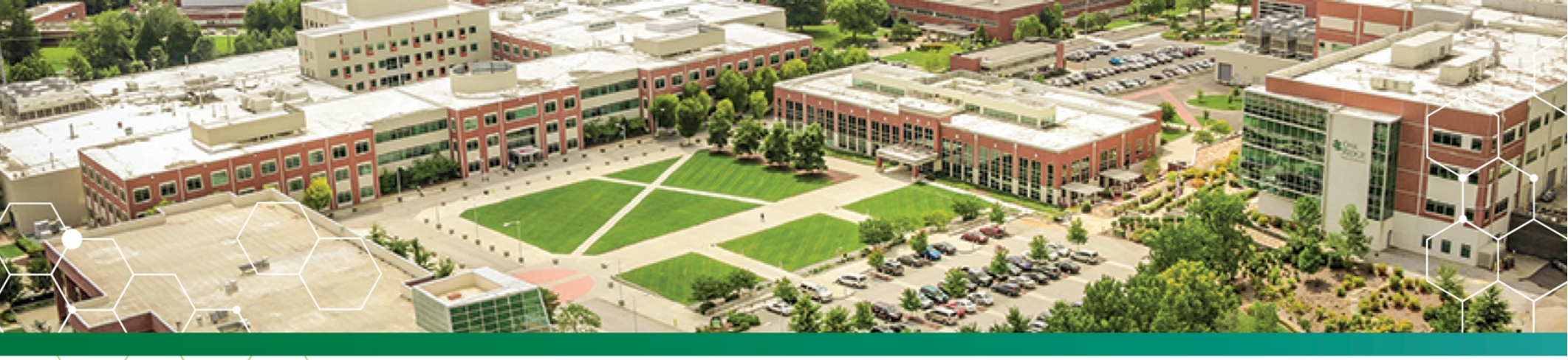

\title{
Impact of Connected Communities
}

\section{Introduction}

Buildings account for $35 \%$ of $\mathrm{CO}_{2}$ emissions and almost $40 \%$ of the United States' energy use. ${ }^{1}$ High-performance homes and neighborhoods play an important role in supporting efforts to decarbonize the US power system by 2035. Significant reductions in $\mathrm{CO}_{2}$ emissions within the residential sector can be realized through electrification of loads paired with the flexibility created by leveraging smart Internet of Things (IoT) capabilities to shift energy use based on grid signals, thus improving generation/distribution efficiency and maximizing the use of renewable generation capacity. All of this can be achieved while allowing smart home appliances and equipment to meet homeowner needs - including reducing power bills - while optimizing operation in conjunction with the grid using novel control techniques. The Grid-Interactive Efficient Buildings Roadmap ${ }^{2}$ by the US Department of Energy's (DOE's) Building Technologies Office (BTO) notes that implementing grid-interactive efficient building (GEB) technology has the potential to reduce $\mathrm{CO}_{2}$ emissions by 80 million tons/year-roughly equivalent to 17 million cars.

To achieve this vision, the US Department of Energy's Oak Ridge National Laboratory (ORNL) -in collaboration with Southern Company Research \& Development, Alabama Power, Georgia Power, BTO and the US DOE's Office of Electricity (OE) -is developing and demonstrating novel connected communities at two locations. Southern Company in turn engaged with industry partners, including design firms, residential developers, and residential HVAC and appliance manufacturers because their participation would be critical to the success of the initial research project, as well as the future scaling to the Southern Company service territory and beyond.

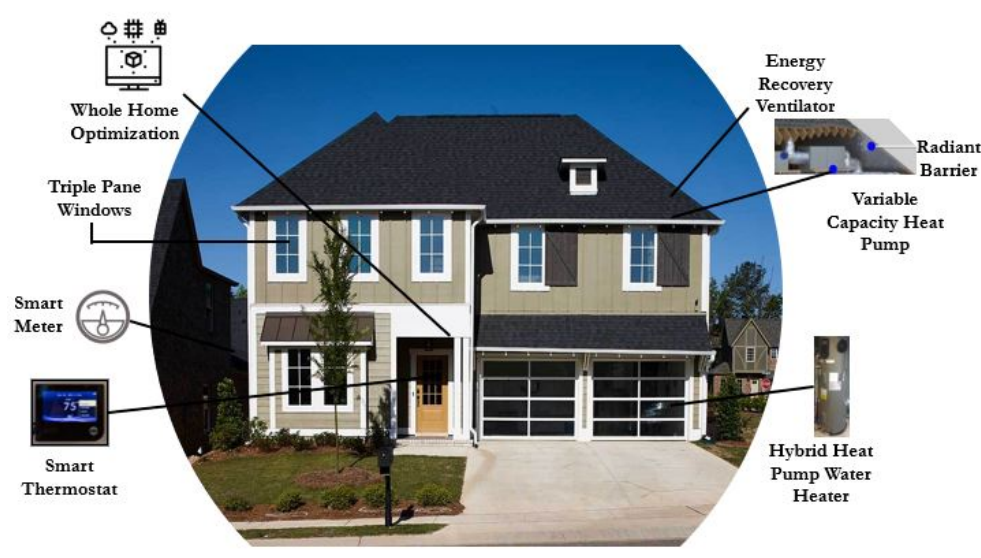

Alabama Power Smart Neighborhood $\AA$ Idea Home. Image courtesy: Alabama Power.
Through its Smart Neighborhood $₫$ program, Alabama Power led the design and development of a community of 62 single-family homes in Hoover, Alabama to include utility-owned, community-scale distributed energy resources (solar, battery storage and backup natural gas generator). The Reynolds Landing at Ross Bridge Smart Neighborhood ( $]$ development is the Southeast's first neighborhood to locally generate, store, and distribute power in conjunction with optimizing and controlling electrical loads within the neighborhood homes. Each home featured enhanced energy efficient components and systems including: $2 \times 6$ walls with blown-in insulation, triple pane low-e windows, attic radiant barrier, hybrid heat pump water heater, variable capacity heat pump, energy data monitoring center, induction cooking, smart outlets and lighting.

\footnotetext{
1 "About the Building Technologies Office," US Department of Energy Office of Energy Efficiency \& Renewable Energy. [Online] Available at https://www.energy.gov/eere/buildings/about-building-technologies-office.

${ }^{2}$ A. Satchwell et al., A National Roadmap for Grid-Interactive Efficient Buildings, Lawrence Berkeley National Laboratory, 2021. [Online] Available at https://gebroadmap.lbl.gov/. See also https://www.energy.gov/eere/buildings/grid-interactive-efficientbuildings.
} 
Southern Company and Georgia Power developed the first Smart Neighborhood $\mathbb{R}$ in Atlanta: Altus at the Quarter. The Altus neighborhood consists of 46 townhomes. In addition to energy efficiency upgrades in the form of high-efficiency heat pumps, smart thermostats, and hybrid heat pump water heaters, each townhome is also equipped with a rooftop solar power system, an inverter, two home energy storage batteries, and an electrical vehicle charger. Each home also includes a transfer switch and critical load panel to allow important loads in the home (refrigeration, select lighting and receptacles) to be powered from the home's batteries in the event of a grid outage.

\section{Learnings: Energy, Grid Services, and Data Management}

\section{Energy}

Based on analysis of annual energy data collected by ORNL, Southern Company, Alabama Power, and Georgia Power, both neighborhoods were substantially more energy-efficient than similar neighborhoods of all-electric homes that had been built to code minimums. Baseline performance of both neighborhoods was compared with similarly sized homes that were constructed to code minimum standards.

Data collected in 2019 showed that Reynolds Landing homes used 44\% less electricity than the baseline neighborhood ${ }^{3}$. In 2020 optimization control of the homes was suspended temporarily during the period of the COVID-19 related "Safer at Home" order in Alabama. Data collected during this time provided insight into the usage patterns for home equipment and appliances and showed the continued benefits of the higher efficiency home design and construction. Comparing similar weather days in 2019 and 2020 showed that both the Reynolds Landing and the baseline neighborhood used more energy in 2020. More detailed evaluation of the circuit level energy use in the Reynolds Landing homes showed that during the "Safer at Home" order, certain loads (range, clothes washer, clothes dryer and water heater) used more energy as compared to the same time in 2019. The analysis also showed that daytime usage peaks occurred later in the morning and earlier in the evening. Even with the increased energy use by household appliances and equipment, the Reynolds Landing daily energy use in 2020 was still $21 \%$ lower than the baseline neighborhood.

Utility meter data and solar generation data from Altus homes shows that on average the Altus homes purchased $42 \%$ less electricity from Georgia Power than the homes in a similar baseline neighborhood ${ }^{4}$. Excluding solar generation, the Altus homes used $20 \%$ less energy than the baseline neighborhood. This compares well with the original energy modeling for the neighborhood that projected $23 \%$ energy savings and $39 \%$ less energy purchased with solar generation included.

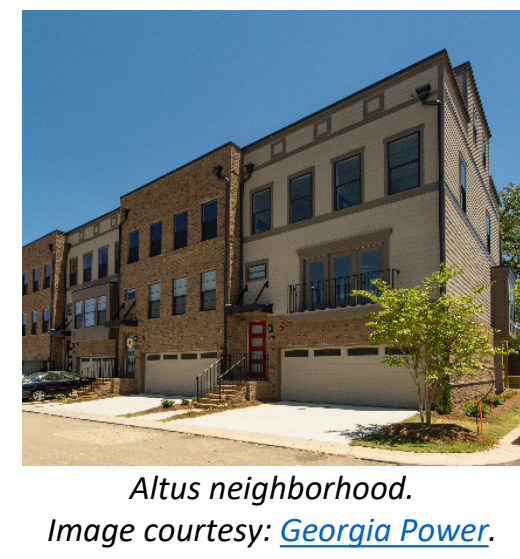

RESULTS

Reynolds Landing 2019

$44 \%$ less energy consumed than baseline neighborhood

7,167 kWh saved annually per home on an equivalent square foot basis

$\$ 931$ saved annually per home on an equivalent square foot basis

5.6 tons of $\mathrm{CO}_{2}$ generation annually avoided per home

Altus 2020

$42 \%$ less energy purchased annually compared with baseline

neighborhood on sq ft basis

$20 \%$ energy savings (excluding PV generation) compared to baseline neighborhood on sq $\mathrm{ft}$ basis

Homes averaged 873 kWh sold back to Georgia Power annually

In winter, 30\% lower maximum hourly $\mathrm{kW}$ demand than baseline

In summer, $62 \%$ lower maximum hourly kW demand than baseline

9.3 tons of $\mathrm{CO}_{2}$ generation annually avoided per home

\footnotetext{
3 "Baseline neighborhood" for the Alabama project is a nearby neighborhood built the same year as the Reynolds Landing homes, with all-electric appliances and HVAC and constructed to code minimum requirements of the 2015 Alabama Energy Code.

4 "Baseline neighborhood" for the Georgia project is a nearby townhome development having the same floorplans as the Altus homes, with all-electric appliances and HVAC and constructed to code minimum requirements of the 2009 International Energy Code (with 2012 Georgia amendments).
} 


\section{Grid Services}

In terms of grid services, the ORNL Oak Leaf optimization and dispatch system shaped and shifted loads by changing HVAC, water heater, and inverter set points ahead of real-time price increases forecast by the utility.

At the Reynolds Landing neighborhood, HVAC peak power demand reductions of $15 \%$ were observed in summer operation, and water heater demand was reduced $43 \%$ in winter operation.

The ORNL Oak Leaf optimization/dispatch showed $12 \%$ demand $(\mathrm{kW})$ reduction at the Altus neighborhood by precooling the homes in advance of an afternoon price peak. Testing is ongoing at the neighborhood, and similar or better results are anticipated for peak winter heating conditions.

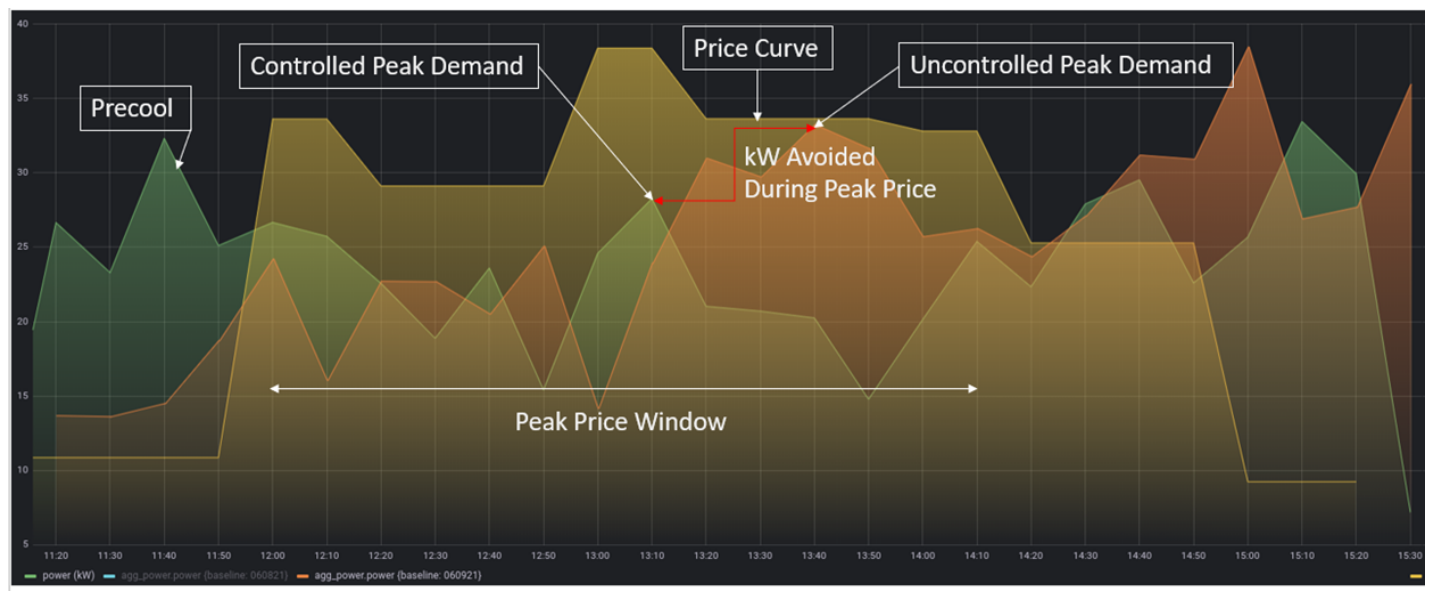

Altus Oak Leaf load shift showing the difference in peak usage during a peak price window (June 2021).

\section{Data Management}

For both neighborhoods, data infrastructure and communications methods were established by Southern Company and ORNL. Data was used to assess the state of devices, inform calculations for optimization, and assess the performance of optimization dispatch versus baseline operation. A layered approach was used to include the data acquisition/storage layer, the analytics layer, and the user interface/visualization layer. The project required development of infrastructure to extract, transform, and load data from

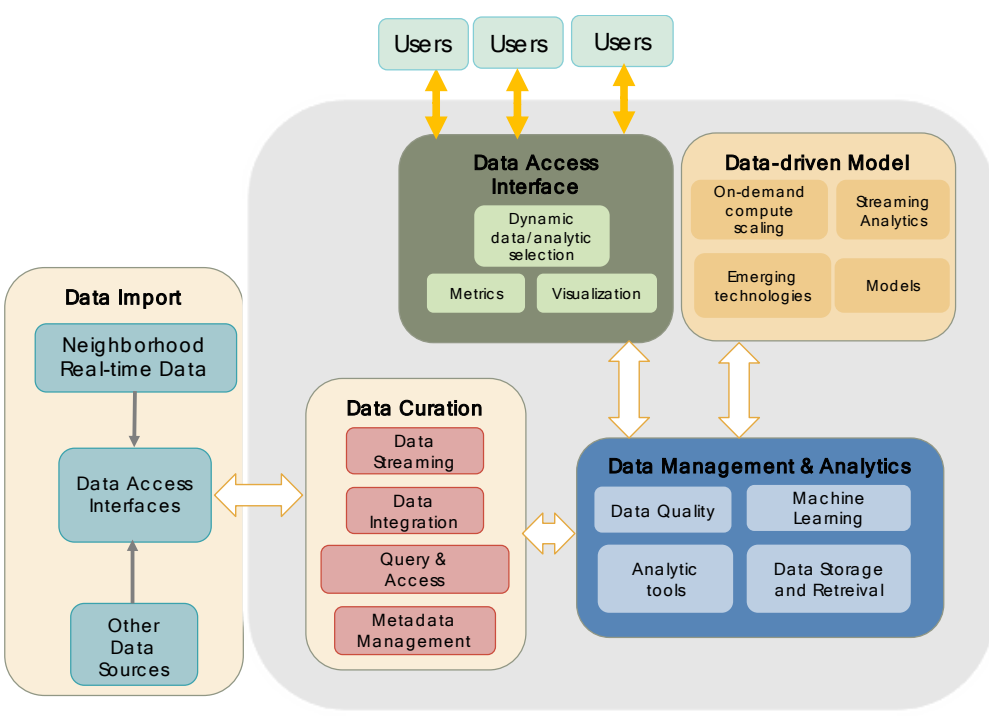

ORNL Data Management Framework. varying sources into specific databases. A framework for data warehousing was created using virtual machines and containers in the cloud. An ecosystem of tools was created to analyze and visualize data. Interactive dashboards were created to monitor system health and rapidly analyze trends using visual clues.

The learnings from the Reynolds Landing project were used to streamline the Altus data management and analysis. Data requirements were established to minimize data translations after acquisition. Automated routines and common databases were used to reduce infrastructure requirements. Flexible architecture was employed with cloud computing to accelerate analytics with elastic computing.

Data has related requirements in terms of confidentiality, integrity, and availability. To harvest data from different platforms and devices, a middleware is needed to abstract 
customer protected information and assist with scaling, without adding latency. In this research project, that functionality was provided by Southern Company's Residential Energy Service (RES) data system. Cloud computing enables data availability, and fault monitoring enables maintaining availability.

\section{Homeowner Feedback}

At the Reynolds Landing neighborhood, Southern Company and Alabama Power conducted monthly homeowner surveys to gauge their opinions on smart home technologies and controls. Location and architectural design were the biggest drivers for buyers to purchase homes in Reynolds Landing, and

\section{The Homeowner's Viewpoint}

"I can make adjustments to ensure my home is well cared for. I can see what my home's electricity usage is with the click of a button, but I can also make any necessary changes due to humidity, temperature, or day-to-day preferences." - Altus homeowner smart home technology was the next most important factor in the purchasing process. Reynolds Landing homeowners reported using voice-controlled home assistants, smart home security systems, and smart lighting controls/receptacles most often.

In Reynolds Landing, $71 \%$ of homeowners reported that they noticed temperature changes as part of the grid-interactive operation of their home HVAC. Of that group, most (65\%) reported that they noticed these changes occasionally each week. This finding indicates how closely the home optimization system followed the preferred temperature ranges for

the home and still provided energy savings through grid-optimized operation.

Homeowners who noticed a temperature adjustment reported that they exercised their option to override the temperature setting for comfort reasons. Homeowners commented that receiving a notification-before making an optimization set point change for energy savings-would be helpful. In addition, about half of Reynolds Landing homeowners suggested that an end-of-month recap of homeowner set point versus optimized set point would be helpful in understanding the set point changes made on their behalf for energy savings.

Altus neighborhood homeowners were asked to identify which of seven smart home technologies was their favorite. $41 \%$ identified the rooftop solar system. Battery storage was the favorite for $18 \%$ of homeowners. Tied for third place with $12 \%$ each were energyefficient smart appliances, voice-controlled home automation systems, and home security systems.

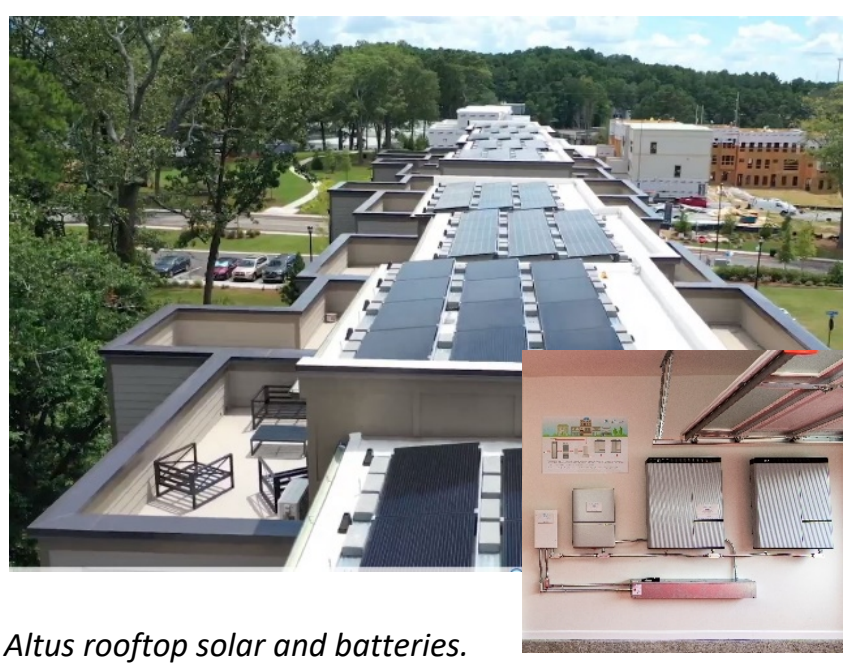

Images courtesy: Georgia Power. 


\section{Vendor Engagement}

Some residential appliance manufacturers offer branded suites of interoperable connected appliances with a common app for customer use. However, no readily available kit or interface seamlessly integrates various appliances with the HVAC, water heater, electric vehicle charger, and/or solar/battery equipment. For appliance manufacturers, offering an interoperable product line provides a sales advantage because builders often purchase a common package of appliances for installation in all homes within a new development.

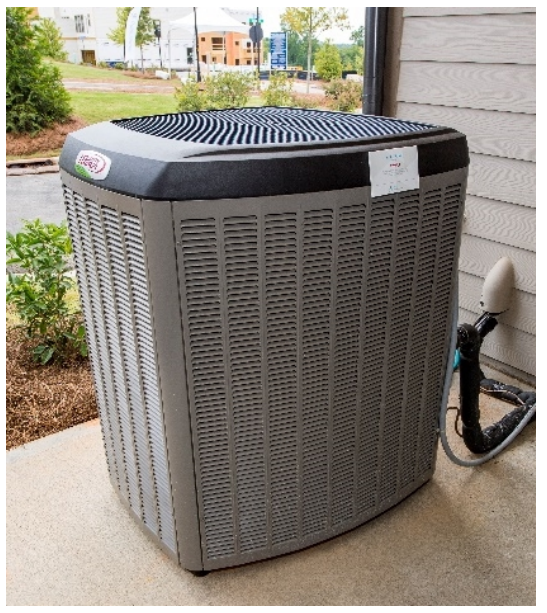

High-efficiency heat pump. Image courtesy: Georgia Power.

For both the Reynolds Landing and Altus neighborhoods, vendor participation was key to the success of the project. This includes manufacturers granting access to their Application Program Interface (API) to allow the Southern Company Residential Energy System to receive device data and pass along optimized device set points from ORNL's Oak Leaf home optimization and dispatch logic. The ORNL team coordinated closely with HVAC, water heater, thermostat, and inverter manufacturers to obtain information about their

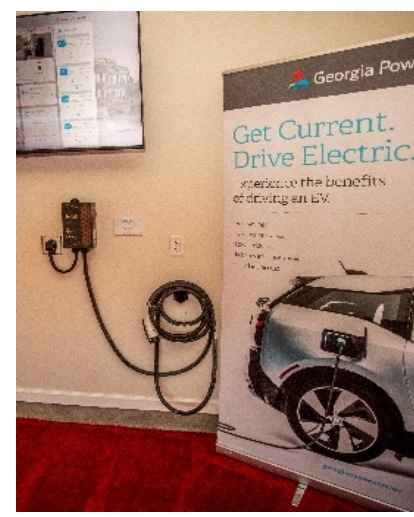

Altus home electric car charger. Image courtesy: Georgia Power. API, device data structure, and control parameters. This collaboration allowed the team to refine the optimization equations, coding, and dispatch control choices to suit each type and brand of equipment.

An example of successful vendor interaction is the inverter manufacturer for the solar photovoltaic systems installed at the Altus neighborhood. In this case, the ORNL team worked with the inverter manufacturer to understand their API, data structure, and control modes available through the API via the Modbus register. A hybrid approach using both the API and Modbus was used to allow information transfer and control dispatch.

Standardizing communication protocol, data framework, and allowable control modes/ranges would streamline this process from an ad hoc arrangement for every make/model of device to a consistent framework for each class of equipment - an example is shown in the hybrid heat pump water heater containing the CTA-2045 communications module. Further deployment of a standards based approach would greatly reduce development and implementation costs for GEB operation. It could also provide benefits to individual manufacturers, who could reduce their development and support costs needed for their current proprietary systems.

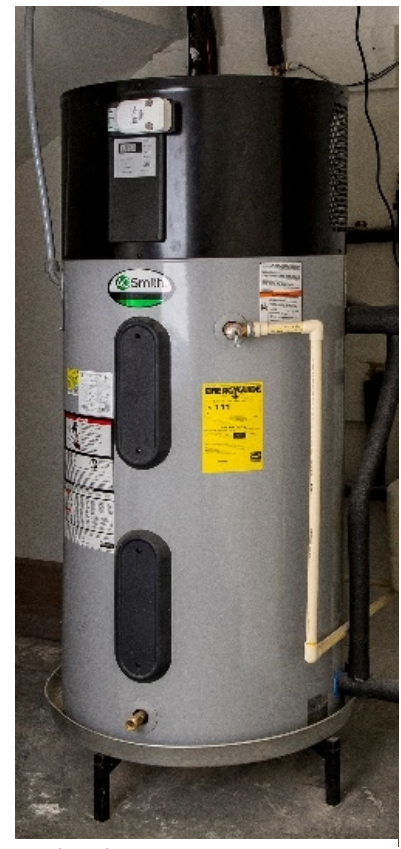

Hybrid Heat Pump Water Heater. Image courtesy: Georgia Power. 


\section{Adoption by Utilities}

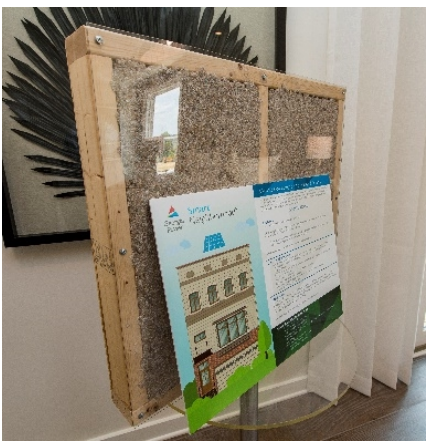

Georgia Power Display of Energy-Efficient Wall. Image courtesy: Georgia Power.
Funded by BTO, ORNL led a virtual workshop in September 2020 with utilities, third-party integrators, and industry consultants to review GEBs, share insights from GEB test cases, identify stakeholder viewpoints, and discuss challenges to GEB adoption at scale. Among the noted challenges were quantifying the value of GEBs both now and in the future (with increasing renewables in the generation portfolio), what implementation would cost, how reliable (e.g., "bankable") GEB benefits are, and how to integrate GEBs with existing energy efficiency and demand response programs. Participants indicated a strong interest in further GEB research and development. Stakeholders also noted that quantifying the value to customers is needed both in terms of energy cost savings as well as non-energy benefits such as improved experience (comfort and resilience). Understanding customer motivations, including interests outside of energy savings, was identified as important to increasing the uptake of smart energy-efficient home technologies and eventually GEBs. Participants agreed that more data is needed to prove the value of GEBs. Scaling up-from pilot-level assessments of residential communities to larger commercial building portfolios and campuses - will be key to proving the GEB opportunity.

\section{Learnings: Technical, Policy, and Business Model}

\section{Technical}

Smart home loads, with the potential to reduce $\mathrm{CO}_{2}$ emissions using grid-interactive controls, include: water heaters, air conditioners, pool pumps, and electric vehicle chargers. ${ }^{5}$

Beneficial electrification of home loads (e.g., shifting from natural gas to electric cooking, water heating, and heating) will bring additional flexible loads into the residential portfolio.

To facilitate implementing grid-interactive controls both Advanced Metering Infrastructure (with its attendant smart meters, communications, and data management) and time-of-use tariff structures need to be in place. Advanced metering uptake has increased greatly since the rollout of the Smart Grid Investment Grant program, funded by the American Recovery and Reinvestment Act of 2009 and administered by the US Department of Energy. As of 2019, some 94 million smart meters were installed nationwide. ${ }^{6}$ Just over $60 \%$ of residences had smart meters. For time-of-use tariffs, the US Energy Information Administration 2019 Annual Electric Power Industry Report Dynamic Price File showed that just over 10 million customers across 700 utilities in the United States were using some form of a time-of-use tariff. ${ }^{7}$ Only $6 \%$ of residential customers were enrolled in some form of TOU tariff. With both advanced metering infrastructure and time-of-use tariffs in place, utilities can work with customers to shift to time-of-use and adopt grid-interactive control of home loads such as the water heater and HVAC thermostats. Utilities such as Duke Power, Holy Cross Energy, Sacramento Municipal Utility District, and Southern Company's Alabama Power and Georgia Power have developed GEB demonstration projects to assess the potential for implementing GEBs across their systems. Research and development on

\footnotetext{
5 "The Smart Home", US Department of Energy Office of Electricity, 2021. [Online] Available at: https://smartgrid.gov/the smart grid/smart home

6 "Frequently asked questions," US Energy Information Administration, 2020. [Online] Available at https://www.eia.gov/tools/faqs/faq.php?id=108\&t=3.
}

7 “Annual Electric Power Industry Report, Form EIA-861 detailed data files," US Energy Information Administration, 2020. [Online] Available at https://www.eia.gov/electricity/data/eia861/. 
communications, data framework/management, and load optimization models is needed to support implementation by third-party demand response integrators who can manage the communication and data management across the utilities' portfolio of customers.

\section{Policy}

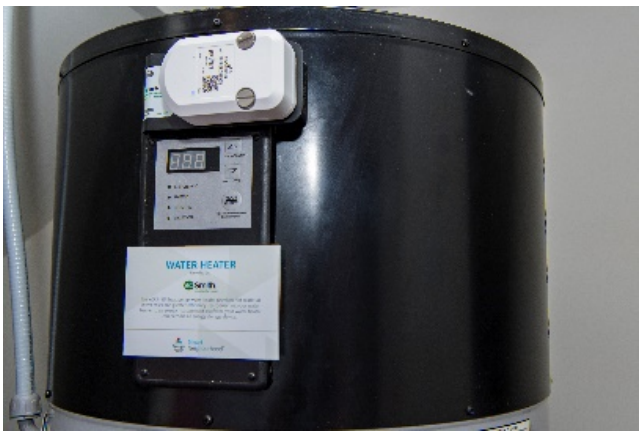

In addition to new technologies, various federal, state and other policy measures could also help increase the adoption of grid-interactive smart home devices and systems for $\mathrm{CO}_{2}$ emission reductions. These advances could include state and federal rulemaking to identify performance criteria, reference standards, testing and certifications, and implementation dates. BTO disseminates regulations for appliance and equipment energy efficiency standards, including test procedures, certification, and enforcement. The US Environmental Protection Agency's voluntary ENERGY STAR and WaterSense programs address appliance and equipment features that provide energy efficiency benefits beyond the minimum standards. The ENERGY STAR program now includes smart devices such as thermostats, lights and switches that

Water heater CTA-2045 module. Image courtesy: Georgia Power. can be incorporated into an ENERGY START Smart Home Energy Management System (SHEMS).

At the state level, California, Washington, and Oregon have been active in developing state polices on grid-interactive appliances and equipment. In 2018, the Washington State Department of Commerce took action to update water heater standards to include aspects such as the adoption of the ANSI/CTA-2045 standard, as well as consumer privacy standards. Policy (rulemaking), codes, and standards actions can all help to standardize device connectivity technology, lower associated hardware and support costs, and move toward interconnectivity between various appliance types and manufacturer makes/models.

\section{Business Model - Smart Home Devices}

Home equipment and appliances only began to feature internet connectivity fairly recently. In a commodity market, appliance and equipment manufacturers likely cannot increase device prices enough to cover the required communications and data support necessary for the 12 to 20 year life of a typical appliance. However, manufacturers can move beyond offsetting the added cost of loT functionality by creating new value from loT-derived data streams. This added value can be generated by using data to inform product enhancements, marketing the data itself as a separate product, optimizing manufacturing processes, improving customer service, and creating new customer experiences. These approaches of using data to add value to the primary equipment and appliances are logical next steps as manufacturers explore loT benefits.

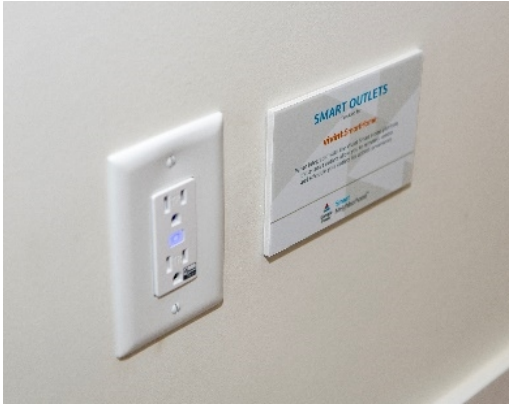

Altus Home Smart Receptacles. Image courtesy: Georgia Power. 